\title{
Pyoluteorin Induces Apoptosis and Autophagy in NSCLC Cells
}

\author{
Daping Fan, Shihuan Yu, ${ }^{*}$ Yue Yang, and Siying Qu \\ Department of Respiratory, First Affiliated Hospital of Harbin Medical University; Nangang district, Harbin, \\ Heilongjiang 150001, China. \\ Received February 7, 2021; accepted April 13, 2021
}

\begin{abstract}
Pyoluteorin is a natural occurring antibiotic and its anti-tumor activity has rarely been reported. This study aims to investigate the anti-tumor effects of pyoluteorin on human non-small cell lung cancer (NSCLC) cells. The cell proliferation was measured by 3-(4,5-dimethylthiazol-2-yl)-2,5-diphenyltetrazolium bromide (MTT) assay. Apoptosis was determined through caspase 3 activity assay and immunoblotting. Autophagy was measured by transmission electron microscope (TEM) and immunostaining. The autophagy-related proteins were detected through immunoblotting. We found that pyoluteorin showed significant anti-tumor effects on human NSCLC cell lines $\mathrm{H1299}\left(\mathrm{IC}_{50}=1.57 \mu \mathrm{M}\right)$ and $\mathrm{H} 2030\left(\mathrm{IC}_{50}=1.94 \mu \mathrm{M}\right)$. Moreover, pyoluteorin could induce apoptosis and autophagy as evidence by the upregulation of caspase 3 activity, the accumulation of LC3 and expression of apoptosis or autophagy related proteins. In addition, pyoluteorin induced autophagy through c-Jun N-terminal kinase/B-cell lymphoma-2 (JNK/Bcl-2) signal pathway. Blocking JNK/Bcl-2 pathway significantly attenuated pyoluteorin-induced autophagy. Moreover, inhibition of autophagy by 3-methyladenine (3-MA) or Beclin1 knockout greatly promoted pyoluteorin-induced apoptosis and cell death. Our results showed that pyoluteorin could induce both apoptosis and autophagy in human NSCLC cells. Combination of pyoluteorin with autophagy inhibitior significantly promoted pyoluteorin-induced apoptosis and may be a potential anticancer strategy in the NSCLC therapy.
\end{abstract} luteorin

Key words autophagy; apoptosis; c-Jun N-terminal kinase (JNK); non-small cell lung cancer (NSCLC); pyo-

\section{INTRODUCTION}

Lung cancer is the most fatal malignancy around the world, with the 5 -year survival rate is about $15 \% .^{1)}$ Histopathological grading identifies more than $85 \%$ of these cases are non-smallcell lung cancer (NSCLC). ${ }^{2)}$ Although the development of different targeted therapy drugs and immunotherapy antibodies, platinum-based chemotherapy is still the standard treatment for NSCLC patients. ${ }^{3-6)}$ However, chemotherapy often causes unpredicted resistance and negative side effects, thus development of novel anti-NSCLC agents is urgently needed.

Pyoluteorin (Fig. 1A), isolated from fluorescent Pseudomonas, is a natural-occurring secondary metabolite and act as an antibiotic and antifungal agent. ${ }^{7)}$ Most research on this compound focus on its antibiotic and antifungal activities. However, its anti-tumor activity has rarely been reported. Recent research showed that pyoluteorin could induce apoptosis in human triple-negative breast cancer cells, ${ }^{8)}$ indicating a critical role of pyoluteorin in cancer therapy.

Autophagy is an evolutionarily conserved process to maintain energy homeostasis through degradation of misfolded proteins and damaged organelles during stress. Many natural compounds could induce both autophagy and apoptosis. However, the triggered autophagy and apoptosis can be synergistic or antagonistic. Natural products like aristolochic acid I could induce autophagy to suppress apoptosis while resveratrolinduced autophagy could trigger cell death thus enhance apoptosis., ${ }^{9}$ ) Therefore, whether autophagy is involved in pyoluteorin-induced cell death and the relationship between autophagy and apoptosis need to be elucidated.

In this study, we showed that pyoluteorin has anti-tumor effect on human NSCLC cells NCI-H1299 and NCI-H2030 by inducing caspase-dependent apoptosis. Pyoluteorin could also induce autophagy by activation of c-Jun N-terminal kinase/B-cell lymphoma-2 (JNK/Bcl-2) pathway. Moreover, autophagy inhibition by 3-methyladenine (3-MA) enhances pyoluteorin-induced apoptosis and cell death in H1299 cells. Our results suggested that combination of pyoluteorin with autophagy inhibitor might be a potential anticancer strategy in the NSCLC therapy

\section{MATERIALS AND METHODS}

Chemicals and Reagents Pyoluteorin with 99\% purity was purchased from APExBIO (Shanghai, China). 3-MA and SP600125 were purchased from Sigma (Shanghai, China). The antibodies were used as follows: caspase3 (\#9662), cleaved caspase3 (\#9661), LC3B (\#3868), p62 (\#5114), Atg5 (\#2630), Atg7(\#8558), Beclin1 (\#3495), pThr183/Tyr183-JNK (\#9251), JNK (\#9252), pSer70-Bcl-2 (\#2827), Bcl-2(\#15071) were from Cell Signaling Technology (Danvers, MA, U.S.A.). Anti- $\beta$-actin was obtained from Santa Curz Biotechnology (Santa Cruz, CA, U.S.A.).

Cell Lines and Cultures Human NSCLC cell line H1299 and H2030 and non-malignant cell BEAS-2B were obtained from cell bank of Chinese Academy of Sciences (Shanghai, China) and maintained in RPMI-1640 medium with 10\% fetal bovine serum and $1 \%$ penicillin-streptomycin. All cell lines were cultured in a humidified incubator at $37^{\circ} \mathrm{C}$ and $5 \%$ of $\mathrm{CO}_{2}$.

Cell Viability Assay cells were seeded in 96-well plates $\left(2 \times 10^{3}\right.$ cells/well $)$ for $24 \mathrm{~h}$ and then treated with indicated concentrations $(0,0.5,1,2,4$, and $8 \mu \mathrm{M})$ of pyoluteorin for $48 \mathrm{~h}$. After incubation, cells were incubated with 3-(4,5-di- 
A<smiles>O=C(c1cc(Cl)c(Cl)[nH]1)c1c(O)cccc1O</smiles>

B

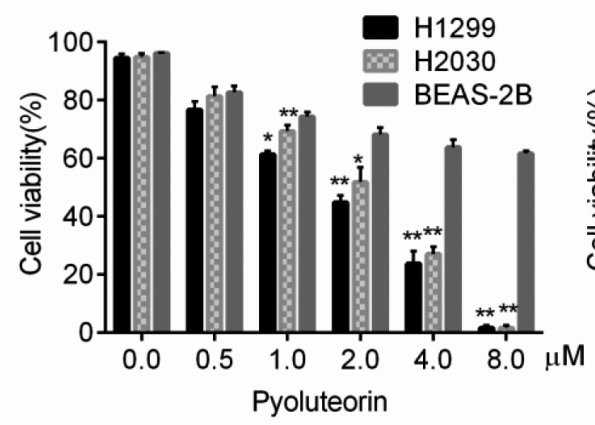

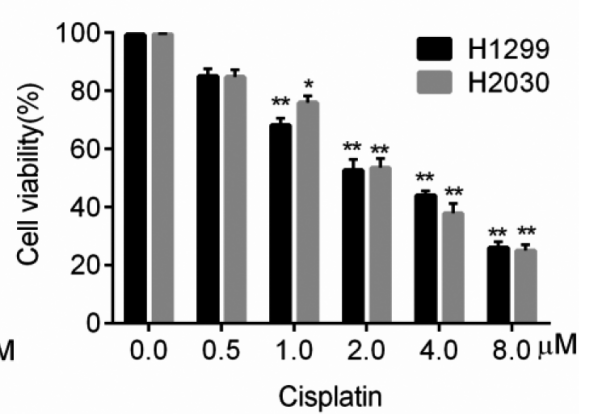

C

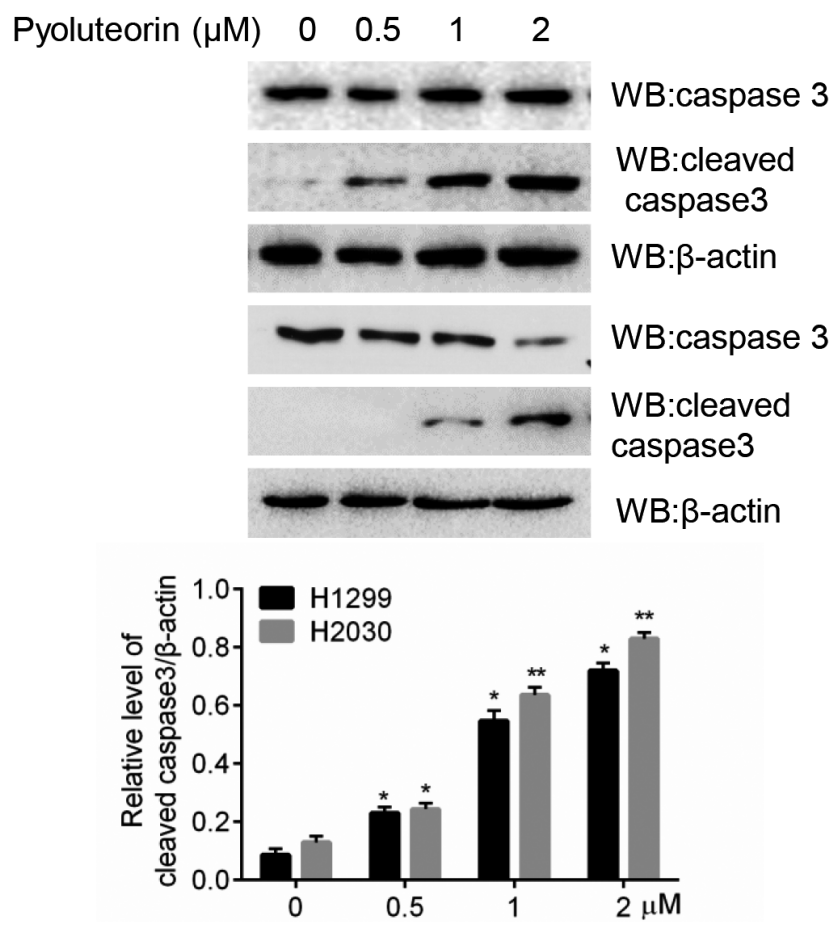

D

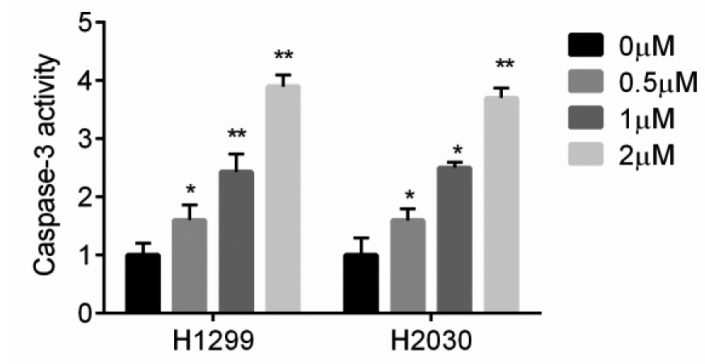

Fig. 1. Pyoluteorin Induces Apoptotic Cell Death in Human NSCLC Cells

(A) The structure of pyoluteorin. (B) NSCLC cells H1299 and H2030 and non-malignant cell BEAS-2B were treated with different concentrations of pyoluteorin or cisplatin and cell viability was performed as indicated. (C, D) H1299 and H2030 cells treated with different concentrations of pyoluteorin as indicated and Western blot (C) or caspase-3 activity assay (D) were performed. Data represent the mean \pm S.D. of triplicate experiments. $* p<0.05, * * p<0.01$.

methylthiazol-2-yl)-2,5-diphenyltetrazolium bromide (MTT) reagent for an additional $4 \mathrm{~h}$ at $37^{\circ} \mathrm{C}$. Cell viability were determined by measuring the absorbance at $570 \mathrm{~nm}$.

Caspase Activity Assay To measure the caspase activity, the Caspase-Glo ${ }^{\mathrm{TM}} 3$ assay kit (Promega, Japan) was used. Briefly, cells were seeded into 96 -well plates $\left(1 \times 10^{5}\right.$ cells/ well) for $24 \mathrm{~h}$. After treated with different concentrations of pyoluteorin for $24 \mathrm{~h}$, the measuring reagent was added to each well and incubated for $1 \mathrm{~h}$ at $37^{\circ} \mathrm{C}$ and then the level of luminescence were measured.

Immunofluorescence Staining After treated with pyoluteorin for 24h, human NSCLC cell line H1299 or H2030 were fixed with $100 \% \mathrm{MeOH}$ and incubated with anti-LC3 antibody at $4{ }^{\circ} \mathrm{C}$ overnight. Cells were then incubated with fluorescein isothiocyanate (FITC)-conjugated secondary antibody for $2 \mathrm{~h}$ in dark as previously described. ${ }^{11)}$

Western Blot Assay Western blot assay was performed as previously described. ${ }^{12)}$ Briefly, nitrocellulose (NC) membranes were block with $5 \%$ non-fat milk for $2 \mathrm{~h}$ and then incubated with indicated primary antibodies overnight. After washing for three times, the membrane was incubated with the secondary antibodies for $1 \mathrm{~h}$.

Transmission Electron Microscopy (TEM) After treated with pyoluteorin for $24 \mathrm{~h}$, human NSCLC cell line H1299 were harvested and fixed for $2 \mathrm{~h}$. Cells were then post-fixed with osmium tetroxide and embedded in EMbed 812 (Electron Microscopy Sciences, PA, U.S.A.). After sectioned and stained, the samples were examined by using JEM-1200EX transmission electron microscope (JEOL, Japan) as previously reported. ${ }^{13)}$

Beclin1 Knockout H1299 or H2030 Cells Clustered regularly interspaced short palindromic repeats CRISPRassociated proteins 9 (Crispr/Cas9) method was used to generate Benclin1 knockout H1299 or H2030 cells as previously 
A
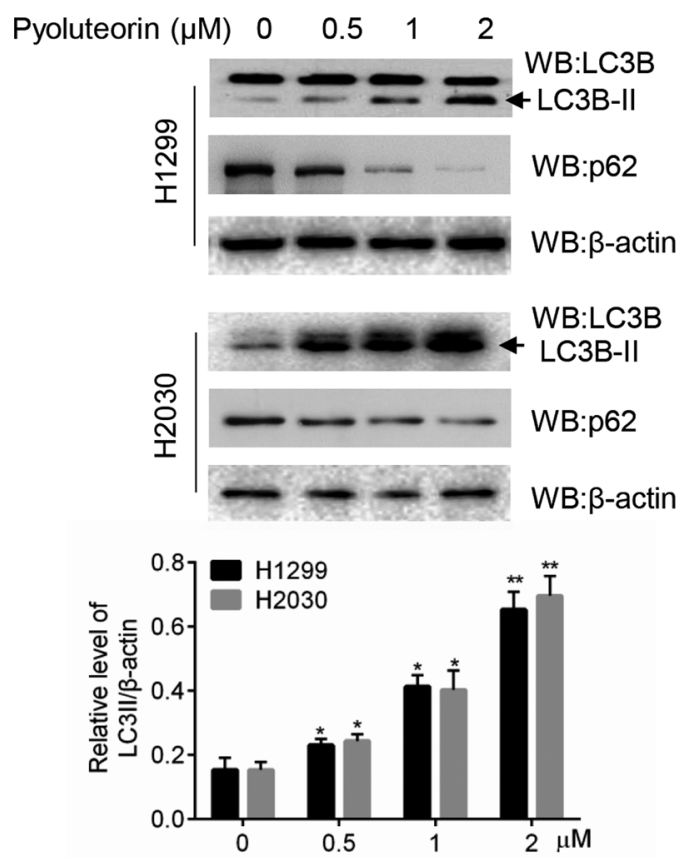

C
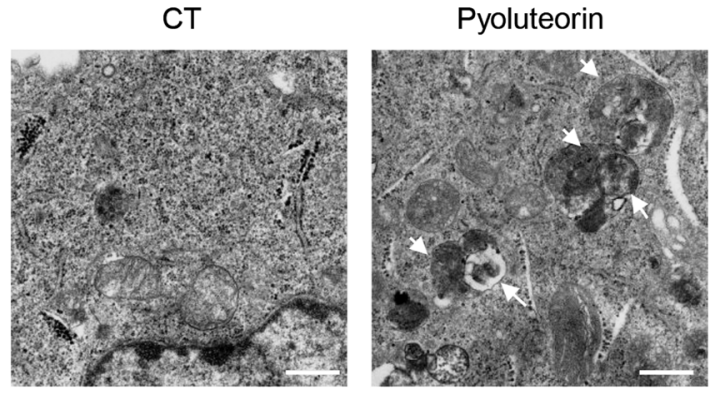

$\mathrm{H} 1299$
B

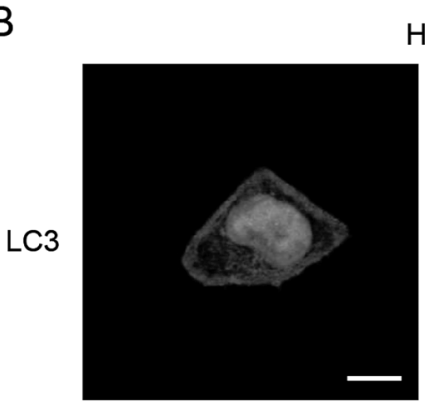

CT

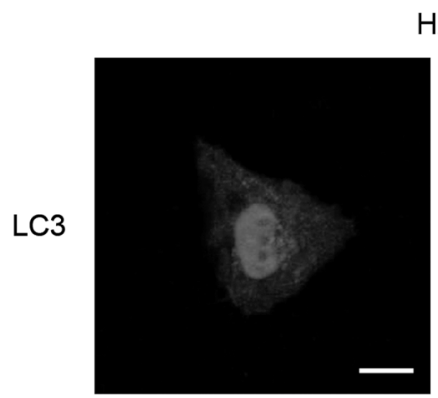

CT
H1299

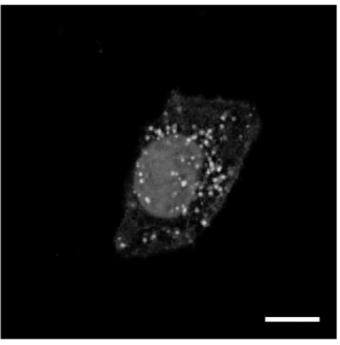

Pyoluteorin

2030

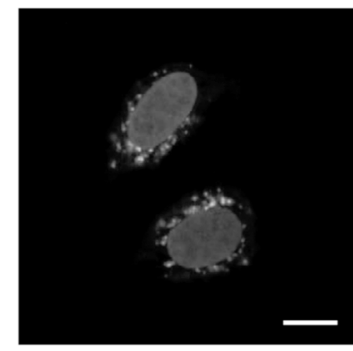

Pyoluteorin
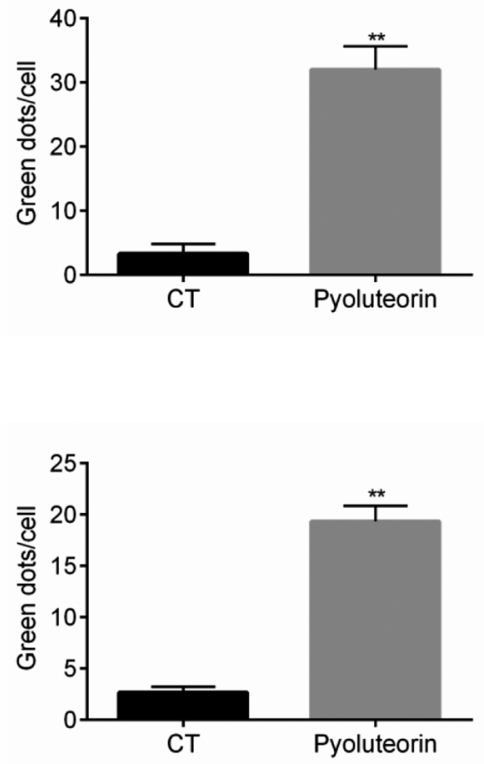

Fig. 2. Pyoluteorin Triggers Autophagy in NSCLC Cells

(A) H1299 and H2030 cells treated with different concentrations $(0,0.5,1,2 \mu \mathrm{M})$ of pyoluteorin were used for immunoblotting. (B) H1299 and H2030 cells treated with or without pyoluteorin $(1 \mu \mathrm{M})$ were used for immunostaining with anti-LC3 antibody. The nuclei were stained with DAPI. (Scale bar $=10 \mu \mathrm{m})$ and the statistics of dark gray puncta per cell were quantified. (Gray: DAPI; Dark gray: LC3B). (C) H1299 cells treated with or without pyoluteorin (1 $\mu$ M) were used for TEM assays (Scale $\mathrm{bar}=500 \mathrm{~nm}$ ). Autophagosomes and autolysosomes (Short white arrows). Data represent the mean \pm S.D., $n=10 . * p<0.05, * * p<0.01$.

described. ${ }^{14)}$ Beclin1 knockout cell lines were generated by using a lentiCRISPRv2-Beclin1 plasmid (5'-CACCGATCT GCGAGA GACACCATCC-3') purchased from Addgene (\#99574). After co-transfected with plasmids (pVsvg : psPAX2 : lentiCRISPRv2-Beclin $1=1: 3: 4)$ in $293 \mathrm{~T}$ cells by using Lipofectamine2000 (Invitrogen, MA, U.S.A.), the recombinant lentivirus encoding lentiCRISPRv2-Beclin1 were used to transduce H1299 or H2030 cells. The stable cell lines were established by selection with $2 \mu \mathrm{g} / \mathrm{mL}$ of puromycin (Invitrogen).
Flow Cytometric Analysis Cells were harvested and washed three times with phosphate buffered saline (PBS). Then cell suspended in binding buffer and incubated with annexinV-FITC and propidium iodide (PI) at $2-8{ }^{\circ} \mathrm{C}$ for $10 \mathrm{~min}$ avoiding light. After incubation, cells were analyzed by flow cytometry (BD PharMingen, CA, U.S.A.).

Co-immunoprecipitation After treatment with pyoluteorin and 3-MA for $24 \mathrm{~h}$, cells were harvest and lysate with $1 \times$ IP lysis buffer. Cell lysate were immunoprecipitation with 
A
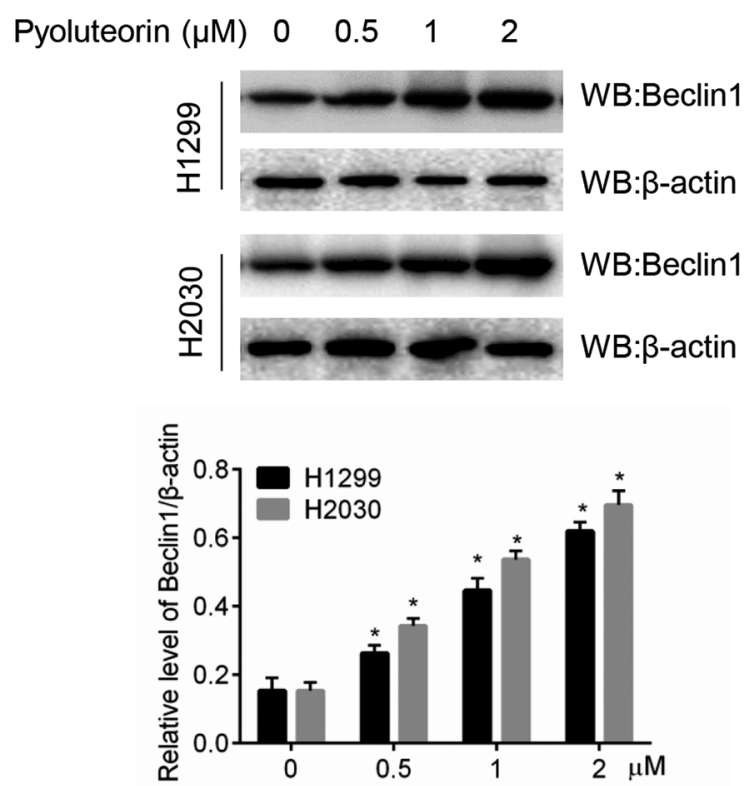

$\mathrm{B}$

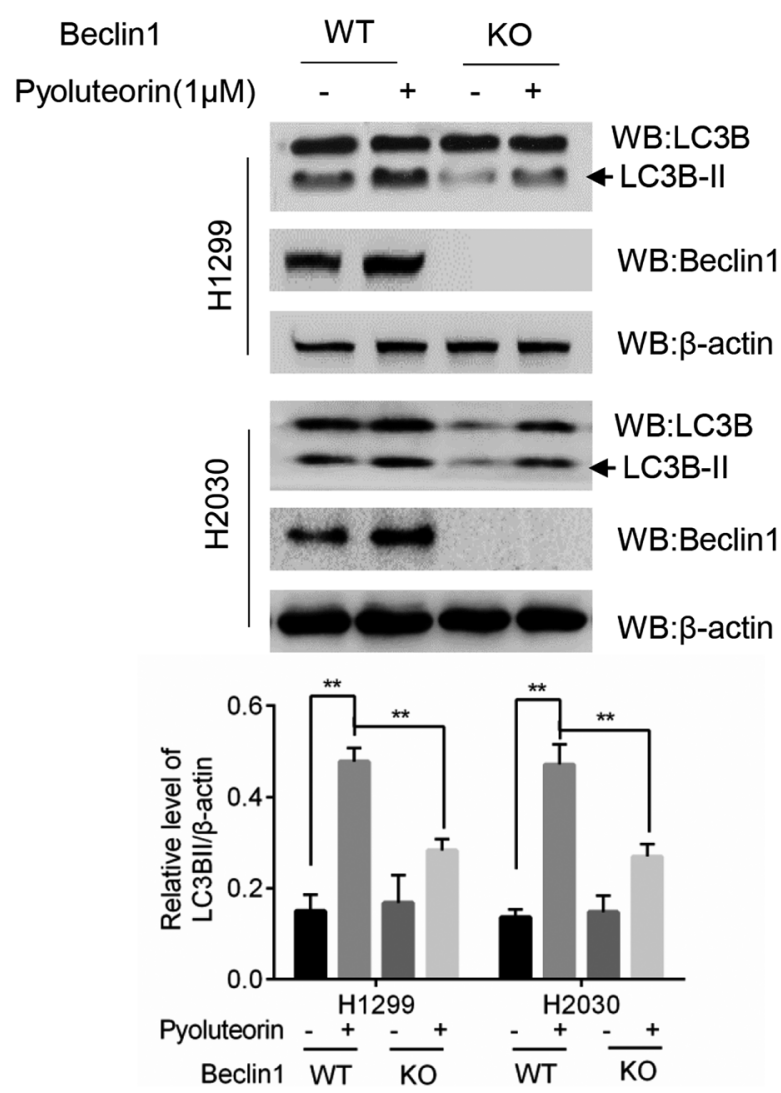

Fig. 3. Pyoluteorin Induces Beclin1 Dependent Autophagy

(A) After H1299 and H2030 cells treated with different concentrations of pyoluteorin for $24 \mathrm{~h}$, autophagy-related proteins were determined by immunoblotting. (B) Beclin1 WT or KO cells were treated with pyoluteorin for $24 \mathrm{~h}$ and immunoblotting assay was performed. All the results were measured three times. Data represent the mean \pm S.D. $* p<0.05, * * p<0.01$.

$1 \mu \mathrm{g}$ anti-Bcl2 antibody for $2 \mathrm{~h}$ at $4{ }^{\circ} \mathrm{C}$. Then the protein $\mathrm{A} / \mathrm{G}$ agarose (Santa Cruz) was added to the lysates and incubated overnight at $4{ }^{\circ} \mathrm{C}$. The immune-complexes were subsequently washed three times with lysis buffer and subjected to immunoblotting analysis as indicated.

Statistical Analyses All experiments were repeated at least 3 times and data were presented as mean \pm standard deviation (S.D.). The difference among treatment groups were analyzed by ANOVA. $p<0.05$ considered as statistically significant.

\section{RESULTS}

Pyoluteorin Induces Apoptotic Cell Death in Human NSCLC Cells We first evaluated the anti-cancer effect of pyoluteorin in human NSCLC cell lines by MTT assay. NSCLC cell lines H1299 and H2030 and non-malignant cell line BEAS-2B were treated with different concentrations of pyoluteorin for $48 \mathrm{~h}$ and cisplatin as positive control. As shown in Fig. 1B, pyoluteorin significantly inhibited H1299 and $\mathrm{H} 2030$ cell viability in a dose dependent manner while the non-malignant cell BEAS-2B was not affected much. Moreover, the growth inhibition efficacy of pyoluteorin to H1299 and H2030 cells were stronger than that of cisplatin. Pyoluteorin had marginally more anti-proliferation effects on $\mathrm{H} 1299$ cells with an $\mathrm{IC}_{50}$ value of $1.57 \mu \mathrm{M}$ than on $\mathrm{H} 2030$ cells with the $\mathrm{IC}_{50}$ value of $1.94 \mu \mathrm{M}$ after $48 \mathrm{~h}$ treatment.

Pyoluteorin was reported to induce apoptosis in breast cancer cells MDA-MB-231. ${ }^{8)}$ To investigate whether pyoluteorin suppress NSCLC cell proliferation through apoptosis, we performed immunoblotting assay. The result showed that pyoluteorin significantly increased the expression level of cleaved caspase 3 (Fig. 1C). In addition, the caspase 3 activity also elevated after pyoluteorin treatment (Fig. 1D). Together, these results suggested that pyoluteorin induces apoptosis in NSCLC cells.

Pyoluteorin Triggers Autophagy in NSCLC Cells To investigate whether pyoluteorin induces autophagy in NSCLC cells, we first measured the expression of LC3BII by immunoblotting. As shown in Fig. 2A, the accumulation of LC3BII increased significantly in a dose-dependent manner after pyoluteorin treatment; while the expression level of p62, an autophagy marker, was down-regulated. In addition, immunostaining assay showed that pyoluteorin treatment obviously increased the number of green puncta of LC3B as compared with control cells (Fig. 2B). Moreover, TEM images showed the accumulated autophagosomes and autolysosomes were observed in pyoluteorin-treated H1299 cells (Fig. 2C). These results suggested that pyoluteorin also triggers autophagy in NSCLC cells.

Pyoluteorin Induces Beclin1 Dependent Autophagy To further investigate the underlying mechanism of pyoluteorininduced autophagy, we examined autophagy-related proteins in H1299 and H2030 cells. The results showed that protein level of Belin1 was up-regulated after pyoluteorin treatment, indicating that pyoluteorin might induce autophagy through 
A

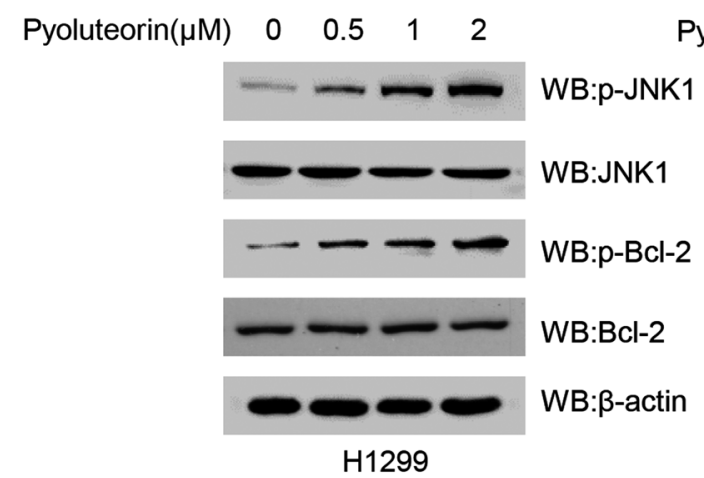

Pyoluteorin $(\mu \mathrm{M}) \quad 0 \quad 0.5 \quad 1 \quad 2$
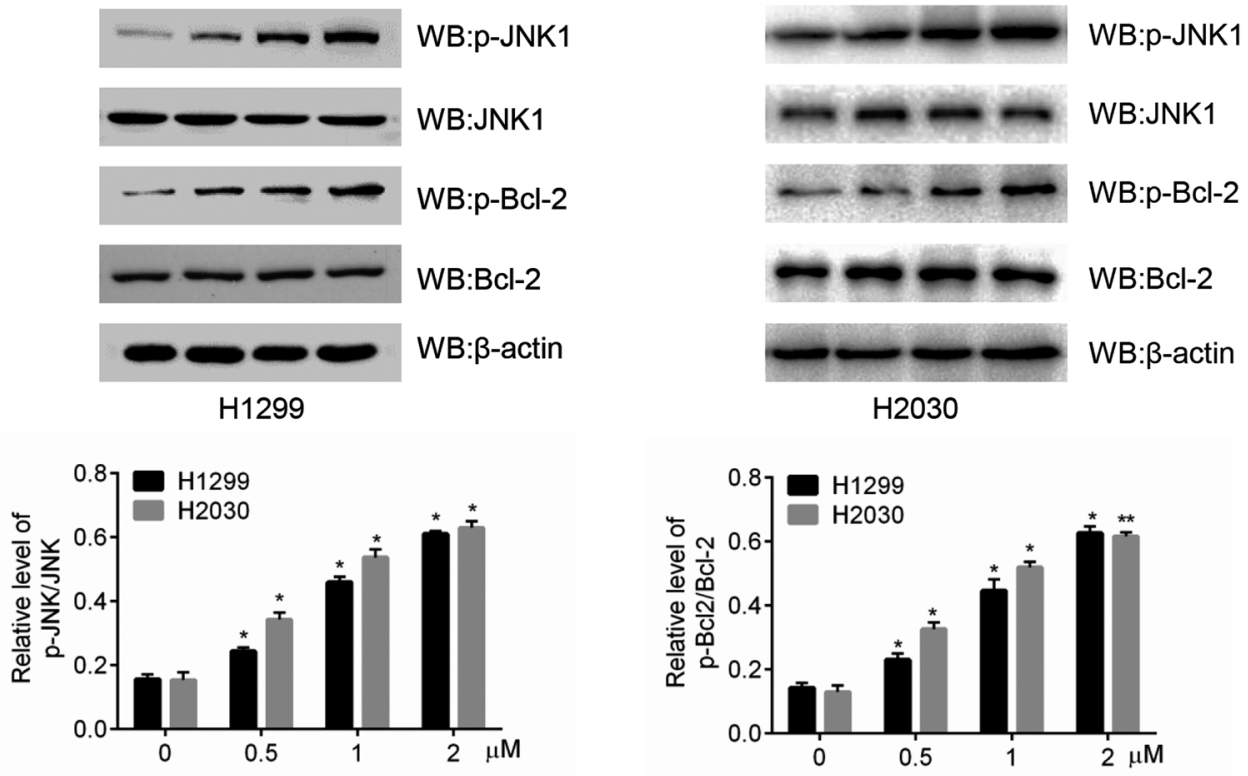

B
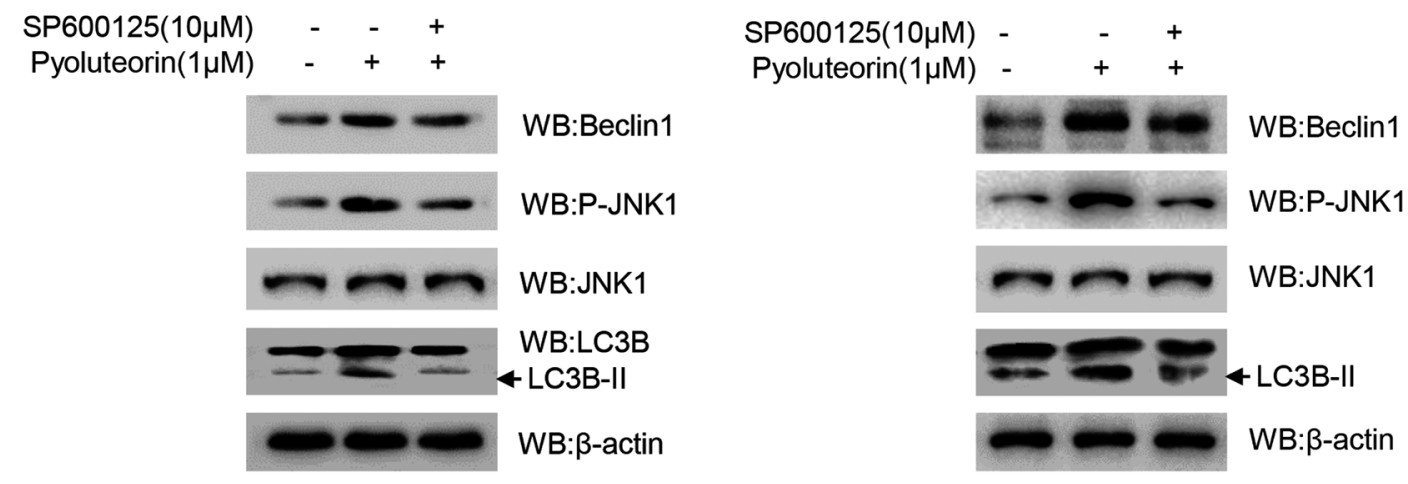

H1299
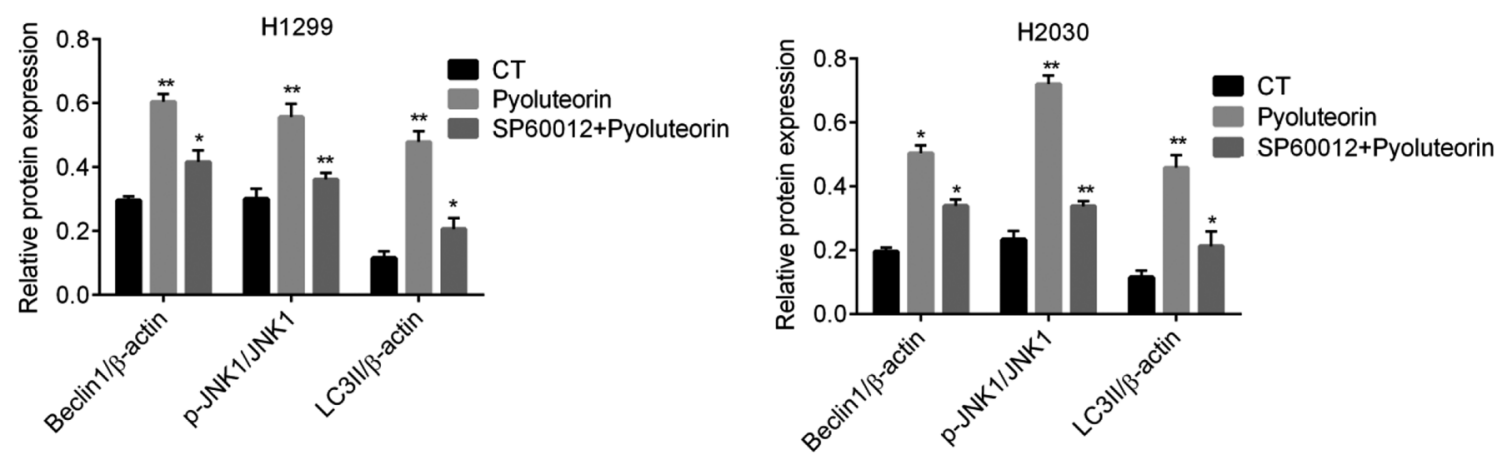

Fig. 4. Pyoluteorin Induces Autophagy through JNK/Bcl-2 Pathway

(A) H1299 and H2030 cells were treated with different concentrations of pyoluteorin for $24 \mathrm{~h}$ and immunoblotting assay were performed as indicated. (B) H1299 and $\mathrm{H} 2030$ cells were treated with pyoluteorin in the presence or absence of SP600125 and immunoblotting assay was performed. All the results were measured three times. Data represent the mean \pm S.D. ${ }^{*} p<0.05,{ }^{* *} p<0.01$.

Beclin1 (Fig. 3A). We further confirmed this result by using crispr/cas9 method to knock out Beclin1 in H1299 or H2030 cells. The results showed that Beclin1 protein expression level was almost undetectable in Beclin1 knock out (KO) NSCLC cells. As expected, pyoluteorin treatment could significantly increase the LC3BII accumulation in Beclin1 wild type (WT) but not in Beclin1 KO H1299 and H2030 cells (Fig. 3B). These results suggested that pyoluteorin-induced NSCLC cell autophagy is Beclin1 dependent.

Pyoluteorin Induces Autophagy through JNK/Bcl-2 Pathway As we known that Bcl-2 interacts with Beclin1 to inhibit autophagy. ${ }^{15)}$ However, JNK phosphorylates Bcl-2 at 


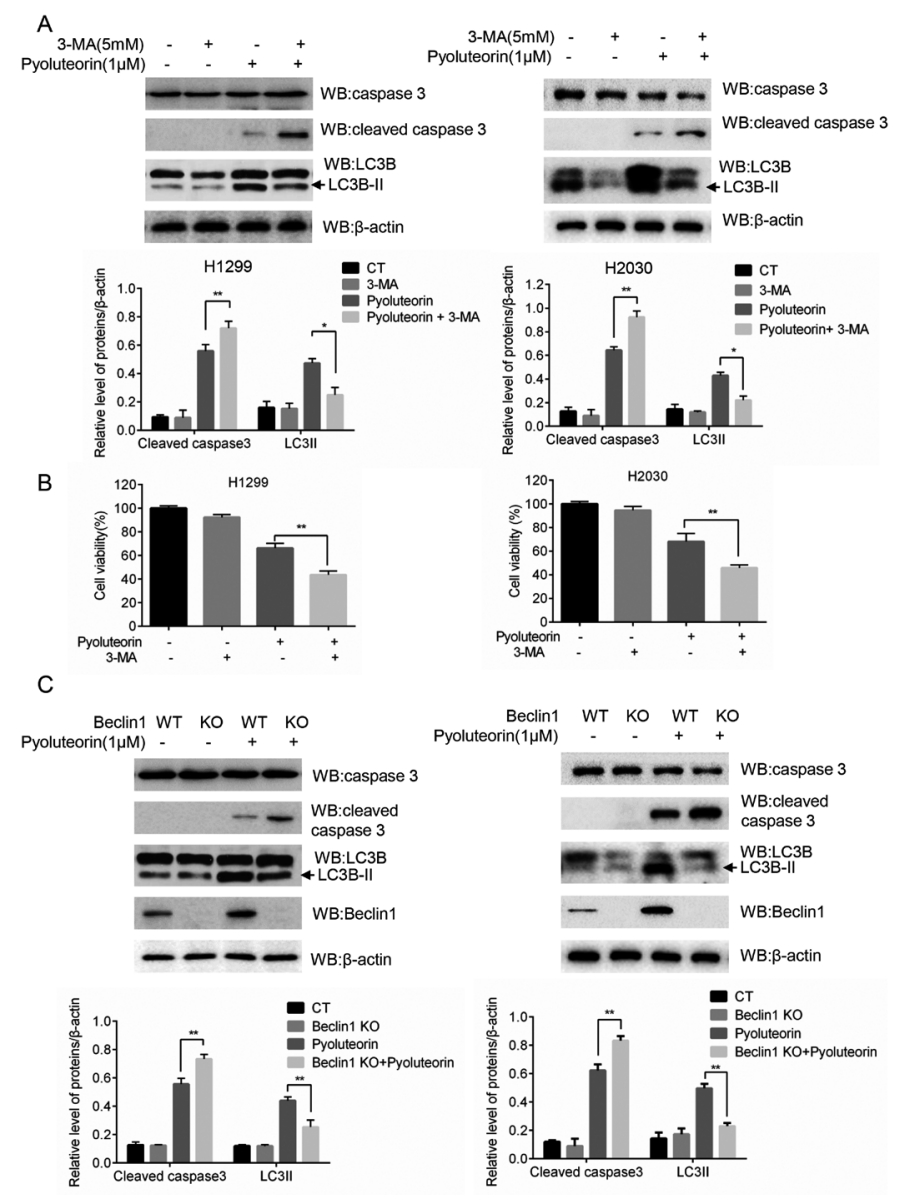

D

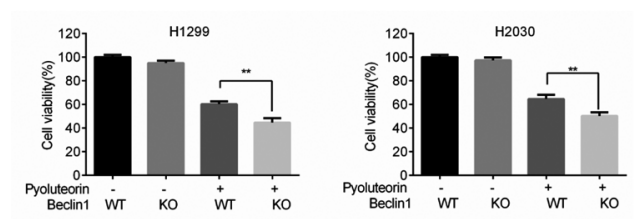

E
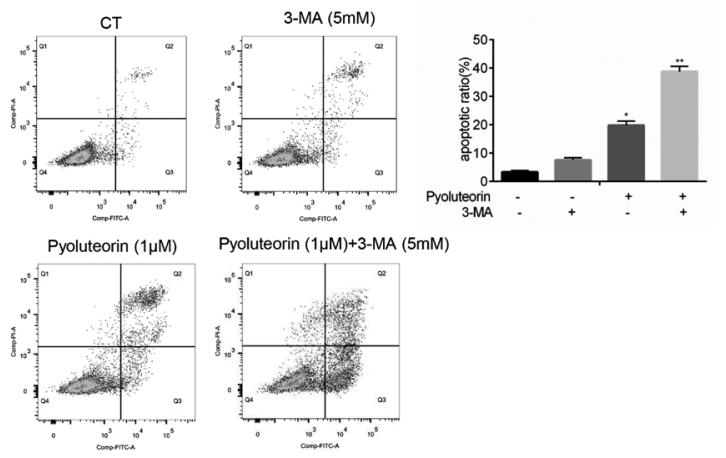

F

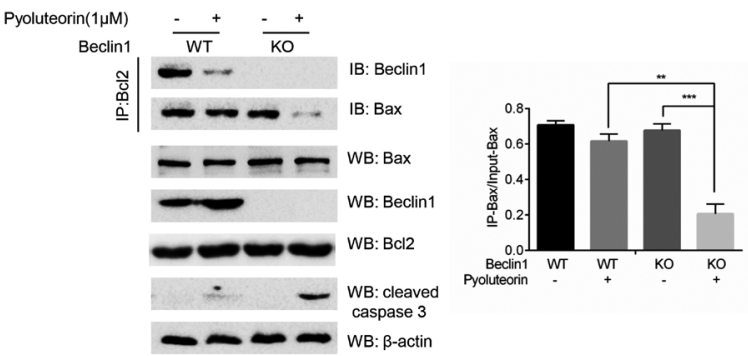

Fig. 5. Pyoluteorin Induces Cyto-Protective Autophagy in H1299 Cells

(A, B) H1299 and H2030 cells were treated with or without pyoluteorin in the presence or absence of 3-MA. Immunoblotting was performed with indicated antibodies (A) and cell viability was measured (B). (C, D) Beclin1 WT or KO H1299 or H2030 cells were treated with or without pyoluteorin. Immunoblotting was performed with indicated antibodies (C) and cell viability was measured (D). (E) H1299 cells were cultured with or without pyoluteorin in the present or absent of 3-MA for 24h and stained by Annexin V and PI staining, then apoptosis rate was determined by flow cytometry. (F) Beclin1 WT or KO H1299 cells were treated with or without pyoluteorin for $24 \mathrm{~h}$. Co-IP was performed with Bcl2 antibody as indicated. Results were represented as the means \pm S.D. of three experiments. $* p<0.05, * * p<0.01$.

S70, thus dissociates Bcl-2 from Beclin1 and leads to the initiation of autophagy. ${ }^{16)}$ We therefore measured the JNK pathway by using Western blot and found that the phosphorylation level of JNK and Bcl-2 were all does-dependently increased after pyoluteorin treatment (Fig. 4A). Moreover, pyoluteorininduced LC3BII and Beclin1 expression were significantly attenuated after SP600125, a JNK inhibitor, added in (Fig. 4B). Together, these results suggested that pyoluteorin induces autophagy through JNK/Bcl-2 pathway.

Pyoluteorin Induces Cyto-Protective Autophagy in NSCLC Cells To investigate the functional role of pyoluteorin-induced autophagy in NSCLC cells, we used 3-MA, an autophagy inhibitor. As expected, 3-MA greatly inhibited the pyoluteorin-induced autophagy as evidence by the reduction of LC3B-I/II conversion (Fig. 5A). However, the combination of pyoluteorin with 3-MA significantly increased the expression level of cleaved caspase 3 as compared with pyoluteorin-treatment alone. In addition, MTT assay showed that the inhibition rate of cell viability was augment in the combination group (Fig. 5B). Pyoluteorin treatment further increased caspase3 cleavage and cell apoptosis in Beclin1 KO but not Beclin1 WT cells (Figs. 5C, D). To further explore the mechanism of how the induced autophagy protected pyoluteorin-mediated apoptosis, we examined $\mathrm{Bcl} 2 / \mathrm{Beclin} 1$ and $\mathrm{Bcl} 2 / \mathrm{Bax}$ interaction by co-immunoprecipitation (Co-IP) assays. The results showed that the interaction of Bcl-2 with Beclin 1 dramatically decreases after pyoluteorin treatment, while the Bcl-2-Bax interaction decreases much slower. However, in Beclin1 depletion cells, the pyoluteorin-induced dissociation of $\mathrm{Bcl}-2$ from $\mathrm{Bax}$ is significantly increased (Fig. 5F). Together, these results suggested that autophagy inhibition could significantly increase pyoluteorin-induced apoptosis in NSCLC cells, indicating that autophagy plays a cyto-protective role in pyoluteorin treated cells.

\section{DISCUSSION}

Platinum-based chemotherapy is indispensable in NSCLC therapy. ${ }^{17,18)}$ However, the drug resistance and negative side effects often occurs within a short time. Therefore, it is urgently needed to develop novel anti-NSCLC agents.

Pyoluteorin, a natural occurring antibiotic, was recently reported to have anti-tumor effects by inducing apoptosis in human breast cancer MDA-MB-231. In this study, we also showed that pyoluteorin has a potential anti-tumor effect on the human NSCLC cells. After treatment with pyoluteorin, we found that the cell viability of NSCLC H1299 and H2030 were significantly inhibited, while the non-malignant cell BEAS$2 \mathrm{~B}$ was not affected much. In line with the findings in breast cancer cells, we demonstrated pyoluteorin could significantly 
induces caspase-dependent apoptosis in NSCLC cells as evidence by the activation of caspase 3 activity and upregulation of cleaved caspase 3 protein level.

Besides apoptosis, many natural compounds could also induce autophagy to enhance or inhibit tumor cell growth. Our results for the first time to demonstrate that pyoluteorin could induce autophagy in NSCLC cells. Both immunoblotting and immunostaining assays showed the significant accumulation of LC3BII after pyoluteorin treatment. However, pyoluteorin could induced LC3BII accumulation in Beclin1 WT but not Beclin1 KO cells, indicating that Belcin1 plays a critical role in pyoluteorin-induced autophagy. Autophagy begins with the formation of autophagosomes, which is regulated by the Beclin1-Vps34 complex to generate phosphatidylinositol3-phosphate (PI3P). ${ }^{19}$ Numerous proteins can interact with Beclin1 to regulate autophagy, which including Bcl-2, an important negative regulator of autophagy. ${ }^{15,16)}$ Phosphorylation of Bcl-2 by JNK can lead to the dissociation of Bcl-2 from Beclin1, therefore promotes autophagy initiation. ${ }^{20)}$ In this study, we found that pyoluteorin could does-dependently increase the phosphorylation of JNK and Bcl-2. In addition, this pyoluteorin-induced up-regulation of Beclin1 and LC3II were significantly attenuated after JNK inhibitor SP600125 added in, indicating that $\mathrm{JNK} / \mathrm{Bcl}-2$ pathway may be indispensable for pyoluteorin-induced autophagy.

Autophagy and apoptosis can be induced by the same stimuli. However, the relationship between them is complicated, as they can affect each other, either synergistically or antagonistically. $^{21,22)}$ Therefore, it is necessary to verify the relationship between pyoluteorin-induced autophagy and apoptosis. Our results demonstrated that pyoluteorin-induced autophagy plays a cytoprotective role in attenuating apoptosis. Inhibition of autophagy by $3-\mathrm{MA}$ or Beclin1 $\mathrm{KO}$ greatly augmented the amount of cleaved caspase 3 and pyoluteorin-induced cell death as compared with pyoluteorin treatment alone. Our results consistent with recent studies showed that natural compounds like paclitaxel, Pharbitis Nil and Paris saponin VII could induce both apoptosis and cyto-protective autophagy in cancer cells. ${ }^{11,12,23)}$

Phosphorylation of Bcl-2 by JNK can also lead to the dissociation of Bcl-2 from pro-apoptotic proteins like Bax to induce apoptosis. Therefore, $\mathrm{Bcl} 2$ phosphorylation may regulate the switch between autophagy and apoptosis, which depends on the dissociation of Bcl-2 from Beclin1 or Bax. ${ }^{20)}$ In this study, we found that $\mathrm{Bcl} 2 / \mathrm{Beclin} 1$ interactions but not $\mathrm{Bcl} 2 / \mathrm{Bax}$ interactions dramatically decreased after pyoluteorin treatment. After Beclin1 depletion, the pyoluteorin-induced dissociation of Bcl-2 from Bax is significantly increased. Taking these results into consideration, we hypothesized that after pyoluteorin treatment, $\mathrm{Bcl} 2$ from $\mathrm{Bcl} 2 / \mathrm{Beclin} 1$ complex was competitively phosphorylated by JNK, leaded to Beclin1 dissociation and autophagy initiation to protect the cell from apoptosis. However, the exact mechanisms still need to be investigated.

Taken together, we demonstrate that pyoluteorin has anti-tumor effect on human NSCLC cells by inducing caspasedependent apoptosis. pyoluteorin could also induce cyto-protective autophagy through the JNK/Bcl-2 pathway in NSCLC cells. Autophagy inhibition promotes pyoluteorin-induced apoptosis. Our findings suggest that pyoluteorin is a potential anti-tumor agent and combination with autophagy inhibitor may be a promising strategy for NSCLC therapy.
Author Contributions SY and DF conceived and designed the experiments; DF, YY and SQ performed the experiments and contributed to molecular analysis; DF and YY analyzed the data; SY wrote the manuscript.

Conflict of Interest The authors declare no conflict of interest.

\section{REFERENCES}

1) Bray F, Ferlay J, Soerjomataram I, Siegel RL, Torre LA, Jemal A. Global cancer statistics 2018: GLOBOCAN estimates of incidence and mortality worldwide for 36 cancers in 185 countries. $C A$ Cancer J. Clin., 68, 394-424 (2018).

2) Soda M, Choi YL, Enomoto M, Takada S, Yamashita Y, Ishikawa S, Fujiwara S, Watanabe H, Kurashina K, Hatanaka H, Bando M, Ohno S, Ishikawa Y, Aburatani H, Niki T, Sohara Y, Sugiyama Y, Mano H. Identification of the transforming EML4-ALK fusion gene in non-small-cell lung cancer. Nature, 448, 561-566 (2007).

3) Tan CS, Cho BC, Soo RA. Next-generation epidermal growth factor receptor tyrosine kinase inhibitors in epidermal growth factor receptor -mutant non-small cell lung cancer. Lung Cancer, 93, 59-68 (2016).

4) Chan BA, Hughes BGM. Targeted therapy for non-small cell lung cancer: current standards and the promise of the future. Transl. Lung Cancer Res., 4, 36-54 (2015).

5) Kanz BA, Pollack MH, Johnpulle R, Puzanov I, Horn L, Morgans A, Sosman JA, Rapisuwon S, Conry RM, Eroglu Z, Johnson DB. Safety and efficacy of anti-PD-1 in patients with baseline cardiac, renal, or hepatic dysfunction. J. Immunother. Cancer, 4, 60 (2016).

6) Maymani H, Hess K, Groisberg R, Hong DS, Naing A, Piha-Paul S, Janku F, Fu S, Tsimberidou AM, Pant S, Karp D, Liu S, Sun M, Heymach J, Simon G, Meric-Bernstam F, Subbiah V. Predicting outcomes in patients with advanced non-small cell lung cancer enrolled in early phase immunotherapy trials. Lung Cancer, 120, 137-141 (2018).

7) Zhang J, Wang W, Lu X, Xu Y, Zhang X. The stability and degradation of a new biological pesticide, pyoluteorin. Pest Manag. Sci., 66, 248-252 (2010).

8) Ding T, Yang L-J, Zhang W-D, Shen Y-H. Pyoluteorin induces cell cycle arrest and apoptosis in human triple-negative breast cancer cells MDA-MB-231. J. Pharm. Pharmacol., 72, 969-978 (2020).

9) Zeng Y, Yang X, Wang J, Fan J, Kong Q, Yu X. Aristolochic acid I induced autophagy extenuates cell apoptosis via ERK 1/2 pathway in renal tubular epithelial cells. PLOS ONE, 7, e30312 (2012).

10) Lang F, Qin Z, Li F, Zhang H, Fang Z, Hao E. Apoptotic cell death induced by resveratrol is partially mediated by the autophagy pathway in human ovarian cancer cells. PLOS ONE, 10, e0129196 (2015).

11) Qian S, Tong S, Wu J, Tian L, Qi Z, Chen B, Zhu D, Zhang Y. Paris saponin VII extracted from Trillium tschonoskii induces autophagy and apoptosis in NSCLC cells. J. Ethnopharmacol., 248, 112304 (2020).

12) Jung HJ, Kang J-H, Choi S, Son YK, Lee KR, Seong JK, Kim SY, Oh SH. Pharbitis Nil (PN) induces apoptosis and autophagy in lung cancer cells and autophagy inhibition enhances PN-induced apoptosis. J. Ethnopharmacol., 208, 253-263 (2017).

13) Chang C, Su H, Zhang D, Wang Y, Shen Q, Liu B, Huang R, Zhou T, Peng C, Wong CC, Shen HM, Lippincott-Schwartz J, Liu W. AMPK-dependent phosphorylation of GAPDH triggers sirt1 activation and is necessary for autophagy upon glucose starvation. Mol. Cell, 60, 930-940 (2015).

14) Huang Z, Fang W, Liu W, Wang L, Liu B, Liu S, Liu S. Aspirin induces Beclin-1-dependent autophagy of human hepatocellular carcinoma cell. Eur. J. Pharmacol., 823, 58-64 (2018). 
15) Maiuri MC, Le Toumelin G, Criollo A, Rain J-C, Gautier F, Juin P, Tasdemir E, Pierron G, Troulinaki K, Tavernarakis N, Hickman JA, Geneste O, Kroemer G. Functional and physical interaction between Bcl-X(L) and a BH3-like domain in Beclin-1. EMBO J., 26, 2527-2539 (2007).

16) Pattingre S, Tassa A, Qu X, Garuti R, Liang XH, Mizushima N, Packer M, Schneider MD, Levine B. Bcl-2 antiapoptotic proteins inhibit Beclin 1-dependent autophagy. Cell, 122, 927-939 (2005).

17) Zeng S, Pottler M, Lan B, Grutzmann R, Pilarsky C, Yang H. Chemoresistance in pancreatic cancer. Int. J. Mol. Sci., 20, 4504 (2019).

18) Hajatdoost L, Sedaghat K, Walker EJ, Thomas J, Kosari S. Chemotherapy in Pancreatic Cancer: A Systematic Review. Medicina (Kaunas), 54, 48 (2018).

19) Backer JM. The regulation and function of Class III PI3Ks: novel roles for Vps34. Biochem. J., 410, 1-17 (2008).
20) Wei Y, Pattingre S, Sinha S, Bassik M, Levine B. JNK1-mediated phosphorylation of $\mathrm{Bcl}-2$ regulates starvation-induced autophagy. Mol. Cell, 30, 678-688 (2008).

21) Bao Y, Ding Z, Zhao P, Li J, Chen P, Zheng J, Qian Z. Autophagy inhibition potentiates the anti-EMT effects of alteronol through TGF- $\beta /$ Smad3 signaling in melanoma cells. Cell Death Dis., 11, 223 (2020).

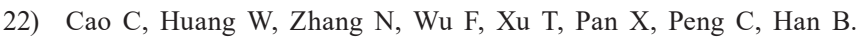
Narciclasine induces autophagy-dependent apoptosis in triple-negative breast cancer cells by regulating the AMPK-ULK1 axis. Cell Prolif., 51, e12518 (2018).

23) Kim HJ, Lee SG, Kim Y-J, Park J-E, Lee KY, Yoo YH, Kim J-M. Cytoprotective role of autophagy during paclitaxel-induced apoptosis in Saos-2 osteosarcoma cells. Int. J. Oncol., 42, 1985-1992 (2013). 